\title{
Quark-Hadron hybrid stars as remnants of Supernovae explosions
}

\author{
Poghosyan, Gevorg S. ${ }^{1}$ \\ Yerevan State University, Alex Manoogian st.1, Armenia 375025
}

It is expected that at explosion of Supernovae the mechanism of producing a shock wave is based on subatomic interactions, and the remnant of the supernovae explosion can be a hybrid quark-hadron star. Since the temperature in the centre of collapsing stars reaches of order $T=6-8 \cdot 10^{10} \mathrm{~K}$ and density $\rho=10^{12}-10^{13} \frac{\mathrm{g}}{\mathrm{cm}^{3}}$, electrons from the top of the Fermi sea can be captured and convert protons into neutrons via $e^{-}+p \rightarrow n+\nu_{e}$. The capture of electrons results in a neutronization burst (V.S.Imshennik 1988). Core collapse of the progenitor star becomes essentially a free fall with a time scale $t \sim \frac{1}{\sqrt{G \rho}} \sim 50 \mathrm{~ms}$. When the central density of the core reaches supernuclear densities the repulsive QCD forces becomes essential. This can bring about manifestations of quarkhadron phase transitions (A.Dar 1997). After the explosion, from the remaining matter is probably formed a hybrid star.

Coming from the afore-mentioned, it is necessary to calculate stable stellar configurations with equations of state (EoS) that take into account quark-hadron phase transitions.I studied and obtained some results for the structure and stability of quark-hadron hybrid stars on the basis of Dynamic, confining (DC) (D.Blaschke 1998) model EoS and Bag model EoS (Ch.Kettner 1995) for quark phase and for hadronic phase. Relativistic nuclear matter EoS including pions and rho mesons was pioneered primarily by J.D.Walecka (J.Walecka 1974). For phase transition construction and mixked phase description, a method is used with more than one conserved charge (N.K.Glendenning 1992).

Figures show differences between hybrid EoS models which arise from mixed phase. The quarks became apparent in Bag model at 2.4 nuclear saturation density and in DC model at 3.2 nuclear density. The second figure shows, that the stable hybrid stellar configurations can exist with masses $2.5-2.6 \mathrm{M}_{\text {sun }}$ with matter described by Bag-Walecka model and $2.8 \mathrm{M}_{\odot}$ by DC-Walecka model. Changes and improvement of EoSes used for quark phase can result in decreasing of masses.

\footnotetext{
${ }^{1}$ post-graduate student at Department of Theoretical Physics, e-mail: gevorg@darss.mpg.unirostock.de
} 


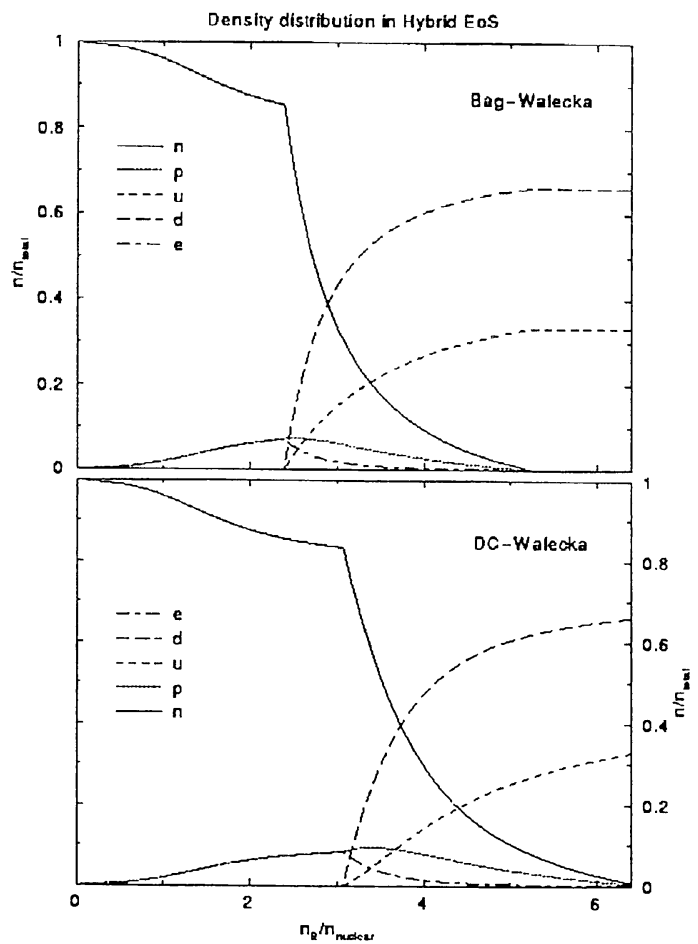

Figure 1. Relative densities depends on baryonic density: n-neutron, p- proton, u-up quark, d-down quark, e- electron 


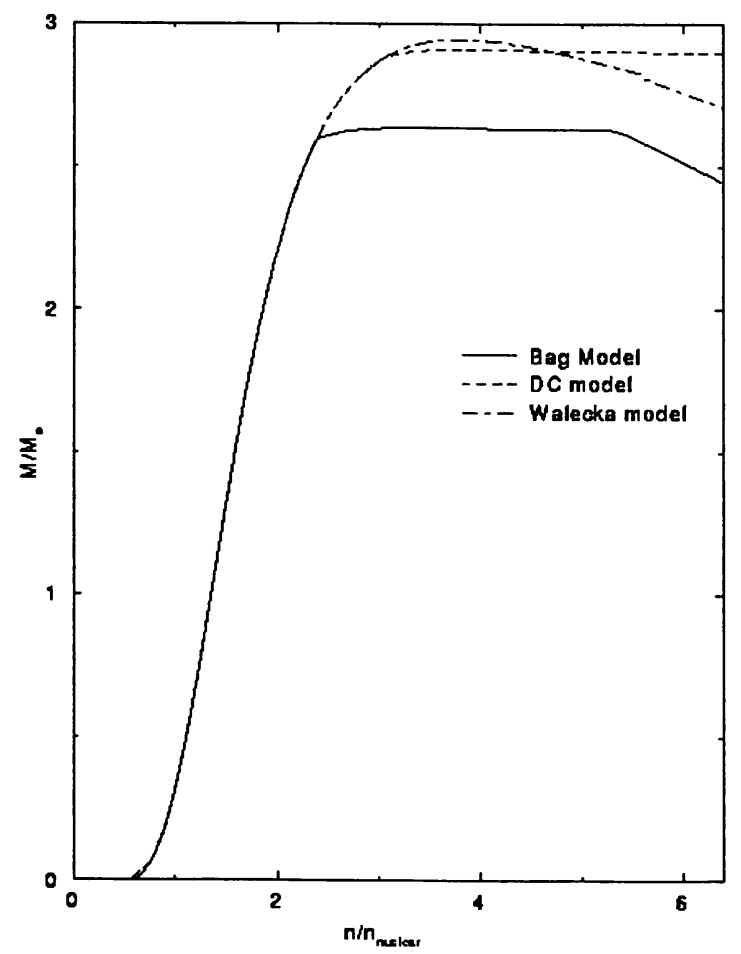

Figure 2. Mass curve of stellar configurations calculated by different EoS. Bag model- by Bag-Walecka hybrid EoS, DC- by dynamic confining-Walecka hybrid EoS, Walecka- pure hadronic EoS

\section{References}

D.Blaschke, C.D.Roberts, S.Schmidt, Phys.Lett.B, 425 (1998) 232 Arnon Dar,"Supernovae 1987A-Ten years after", hep-ph/9707501, N.K.Glendenning, Phys.Rev.D, 46, 1992,1275

V.S.Imshennik, D.K.Nodëzhin, Uspekhi Fizicheskikh Nauk, 1988 v.150, No. 4, 561

Ch.Kettner, F.Weber, N.K.Glendenning, etc Phys.Rev.D 51 (1995), 1440 Walecka, J.D., Ann.Phys., 83,491 (1974) 